\title{
Modified GIANT Dynamic Bandwidth Allocation Algorithm of NG-PON
}

\author{
Tomas Horvath, Student Member, IEEE, OSA, Vaclav Oujezsky, Student Member, IEEE, \\ Petr Munster, Member, IEEE, OSA, Josef Vojtech, Member, IEEE, OSA, Ondrej Havlis, Member, IEEE, OSA, \\ and Pavel Sikora
}

\begin{abstract}
Gigabit passive optical networks have been widely deployed due to the fact that the cost of their implementation is still decreasing. What is more important, we are facing the problem with increasing demands on the transmission bandwidth. Regarding this issue, the ITU develops another two standards supporting higher downstream bitrate. The XG-PON standard is the first platform under the developing, and the NG-PON2 is the second standard. The first one provides compatibility and increases the downstream capacity of $10 \mathrm{Gbit} / \mathrm{s}$ and the second standard has the same assumptions, but does not have backward compatibility. In this article, we discuss only XG-PON networks. We choose amendment as the dynamic bandwidth allocation algorithms, and we have compared it with the original specification and with our modification. The primary intention of that modification is to reduce the delay of Triple Play (data, video, and voice) services. These services are represented by $T$ CONT (Transmission Container), which is used to improve the PON system upstream bandwidth allocation and transmission status dynamically. As NS-3 simulator does not support the direct mapping of Triple Play services into T-CONT and their labeling. We focus on a delay value for Triple Play services which was reduced by own modification in a GIANT algorithm. On the other hand, we cannot reduce the delay value for VoIP services because it has the highest priority by T-CONT.
\end{abstract}

Index Terms-DBA, NS-3, simulation, PON, XG-PON.

\section{INTRODUCTION}

$\mathbf{M}$ Any ISPs (Internet Services Providers) have started to deploy the GPON (Gigabit Passive Optical Network) systems as a reaction on the requirement of the rapidly increasing bandwidth of customers [1], [2], [3]. GPON network supports the high data rate of $2.5 \mathrm{Gbit} / \mathrm{s}$ and of $1.25 \mathrm{Gbit} / \mathrm{s}$ for downstream and upstream. The brief idea of the GPON network is shown in Fig. 1. It is possible to connect up to 64 ONUs (Optical Network Unit) to one splitter. But the bandwidth is not still sufficient for new services or for the increasing resolution of video broadcasting based IPTV (Internet Protocol television) in $4 \mathrm{k}$ or $8 \mathrm{k}$. This creates a need for a technology that supports higher speeds.

ITU (International Telecommunication Union) and FSAN (Full Service Access Network) have started the research of

Manuscript received November 30, 2016; revised February 28, 2017 and March 24, 2017. Date of publication March 28, 2017.

Tomas Horvath, Petr Munster, Josef Vojtech, and Ondrej Havlis are with CESNET a.l.e., Department of Optical Networks, Zikova 4, Prague, Czech Republic (E-mail: horvath, munster, havlis, vojtech@cesnet.cz).

Vaclav Oujezsky and Pavel Sikora are with Department of Telecommunications, Brno University of Technology, Technicka 12, Brno, Czech Republic.

Digital Object Identifier (DOI): 10.24138/jcomss.v13i1.243

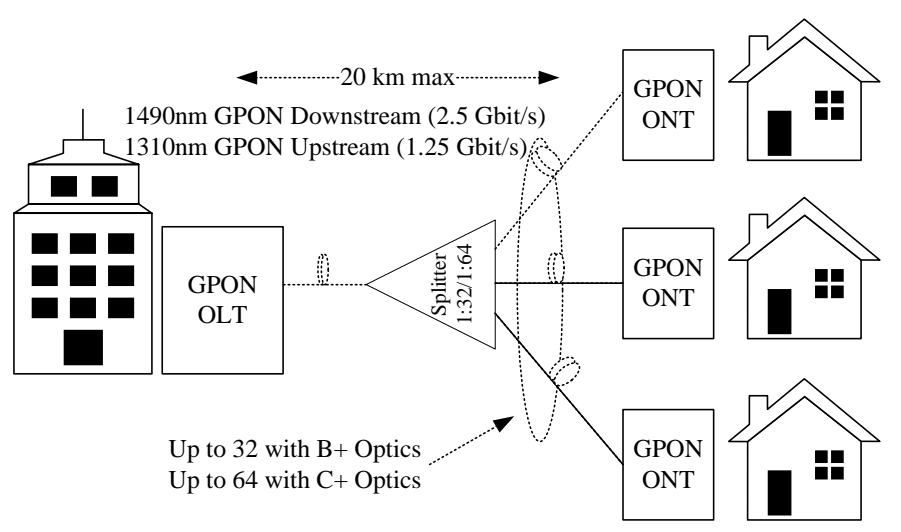

Fig. 1. Basic topology of GPON

a new standard for higher bandwidth. Nowadays, the final specification of XG-PON (Next Generation or 10G Generation) has been approved as ITU-T G.987 series. For example, ITU-T G.987.1 specifies general requirements such as reference configuration of XG-PON, ODN (Optical Distribution Network) architecture (with/without coexistence scheme of previous standards GPON), operating wavelengths, migration scenarios, etc. [4]. The second document of the series deals with the attenuation classes of ODN, fiber distance between OLT (Optical Line Termination) and ONU, line codes for various transmission speed, where NRZ (Non Return Zero) dominates, jitter tolerance, etc. [5]. The following specification ITU-T G.987.3 is the most important for our research, because it specifies transmission convergence layer of XG-PON with the frame structure, frame size, DBA (Dynamic Bandwidth Allocation) algorithm application, all identifiers in the frame, etc. [6].

The basic idea of GPON and the previous standards (ODN structures) were maintained due to the investment protection. The capital assets for ODN comprise approximately $70 \%$ of total investments. That is why ITU and FSAN have to keep the same ODN although OLT and ONU should be replaced. Next generation PON has been divided into two stages. The first stage represents XG-PON with the main aim to keep compatibility with GPON's networks at the same ODN and to increase the downstream rate, at least on $10 \mathrm{Gbit} / \mathrm{s}$. The compatibility is ensured by WDM (Wavelength Division Multiplex) and WDMA (WD Multiple Access) [7]. Note, that XG-PON does not need to support symmetric rate (same 
TABLE I

PARAMETERS OF XG-PON SYSTEMS ACCORDING TO [5]

\begin{tabular}{|l||l|}
\hline \multicolumn{1}{|l|}{ Fiber type } & ITU-T G.652 \\
\hline \hline Optical path loss & $14-29 \mathrm{~dB}$ (N1 class) \\
& $16-31 \mathrm{~dB}$ (N2 class) \\
& $18-33 \mathrm{~dB}$ (E1 class) \\
& $20-35 \mathrm{~dB}$ (E2 class) \\
\hline Maximum fiber distance & $20 \mathrm{~km}$ \\
\hline Split ratio & at least $1: 64$ \\
\hline Downstream wavelength & $1575-1580 \mathrm{~nm}$ \\
\hline Upstream wavelength & $1260-1280 \mathrm{~nm}$ \\
\hline Downstream nominal rate & $9.95328 \mathrm{Mbit} / \mathrm{s}$ \\
\hline Upstream nominal rate & $2.48832 \mathrm{Mbit} / \mathrm{s}$ \\
\hline
\end{tabular}

transmission speed for downstream and upstream). The second stage has the main aim symmetric transmission speed and support of various wavelengths at one ONU. It is the first standard for this purpose. Single ONU can support up to 4 wavelengths with $10 \mathrm{Gbit} / \mathrm{s}$ per lambda from a historical point of view.

Back to the XG-PON, significant parameters of these networks are shown in Tab. I. Study group 15 (the part of ITU which deals with PON development). A suitable fiber can significantly reduce attenuation of ODN. XG-PON considers only single mode (or equivalent with the same or better attenuation).

However, the distance between OLT and ONU can be approximately around $20 \mathrm{~km}$ only (which equals approximately to $4.4 \mathrm{~dB}$ ). The high split ratio leads to restricting the reach of the system. Split ratio there has the same volume as is in GPON but can be higher because the [6] standard specifies ratio at least 1:64 according to attenuation classes. Those are the same with GPON specification. Nominal 1 (14-29 dB), Nominal $2(16-31 \mathrm{~dB})$, Extended $(18-33 \mathrm{~dB})$, and Extended $2(20-35 \mathrm{~dB})$. Note, that ISPs can increase this split ratio or system reach-ability, but it is mandatory to keep total attenuation consistent within attenuation classes or use an optical amplifier in ODN. Furthermore, operational wavelengths in upstream direction overlap GPON upstream but GPON has a wide range of upstream direction, and for coexistence scheme, WDM is used. Downstream wavelength range is only $5 \mathrm{~nm}$ which can lead to the cooling system in OLT for the laser. This adding of the cooling system can increase the cost of the whole system. Wavelength allocation does not consider wavelengths around $1550 \mathrm{~nm}$ because of all PON support IPTV and analog TV type, called CATV (Cable TeleVision). CATV system uses separated wavelengths around $1550 \mathrm{~nm}$, modulation format and type of source depends on ISP, commonly QAM (Quadrature Amplitude Modulation) or OFDM (Orthogonal Frequency Division Multiplexing) are used. More information about CATV system and the deployment in PON can be found in [8], [9]. The transmission speed of the XG$\mathrm{PON}$ is $9.95328 \mathrm{Gbit} / \mathrm{s}$ for downstream and $2.48832 \mathrm{Gbit} / \mathrm{s}$ in upstream.

The main contribution is reducing a time of Triple Play services in XG-PON networks. We have proposed own improvement of GIANT DBA algorithm for $10 \mathrm{Gbit} / \mathrm{s}$ passive optical networks and compared results of unmodified (current) and modified GIANT algorithms. The rest of this paper is structured as follows. In the next section an overview of related works is mentioned. Section 3 presents the GIANT (GigaPON Access Network) algorithm for DBA (Dynamic Bandwidth Allocation) used in XG-PON networks. Section 4 describes a simulation scenario, simulation results with a discussion and represents our improvement specified for decreasing the Triple Play services delay.

\section{RELATED WORKS}

In last few years, many works related to $x \mathrm{PON}$ technology have been published. However, GPON technology is ready for wide deployment of many applications; there are many different standards for PON networks. Researchers mainly focus onto the physical layers of standards although a focus on the transmission convergence layer is also necessary because PON without it will not be able to transfer data. Transmission convergence layer covers coverage of ODN and supports rich extensions. Each standard has to support at least $20 \mathrm{~km}$ per physical distance, but transmission convergence layer allows up to $60 \mathrm{~km}$ due to a longer distance between OLT and ONU, with a higher split ratio, increases attenuation in ODN and it requires optical amplifiers [10][11].

DBA is one of the most important parts of transmission convergence layer. DBA had been firstly used with BPON (Broadband PON) standard, but this solution used ATM (Asynchronous Transfer Mode) cells for transferring data. The following GPON standard wondered the use of ATM cells and Ethernet frame to transfer data, but the latest specification excludes ATM (Asynchronous Transfer Mode) cells transferring. GIANT algorithm has been taken over by XG-PON networks from GPON. In [12] the authors proposed the X-GIANT modification of original GIANT algorithm and considered to reduce the main parameters of QoS (Quality of Services) in XG-PON networks. Furthermore, they focused on the assured and non-assured ratio of medium priority traffic. Their results proved that more allocation cycles increase the total delay of a service. The final usage of essential services (commonly Triple Play services) are missing here.

The work [13] compares available DBA algorithms for XGPON networks such as Iris, GIANT, and SFDBA (Simple and Feasible Dynamic Bandwidth Allocation). The SFDBA algorithm was proposed by Han [14] in contrast to the other two methods; the T-CONT uses the priorities unfairly. It is useful if the traffic, represented by T-CONT 4, is dominating. The article [13] presents the results with average allocation ID delay for every ONU where Iris DBA has the best results, although the 8 ONUs were only taken into account. The real network does not have only 8 ONUs per OLT interface; it can have up to 64 ONUs per OLT interface.

Sarigiannidis et al. [15] presents new DBA scheme for XGPON networks called DAMA (A Data Mining Forecasting). This proposed DBA scheme deals with various distances for each ONU in the span of $40 \mathrm{~km}$ ODN. Note, that it is not such typical distance; however, it is possible to use it if the attenuation classes are taken into account. In general, 
the proposed scheme can forecast the additional bandwidth requirements in ONUs. However, the scheme depends on forecasting algorithm. The OLT has limited resources for all ONUs per OLT port. Standard Triple Play services were tested, and jitter/delay were reduced, but the authors do not count the resources consumption in OLT.

The article [16] introduces another DBA algorithm with the name IFAISTOS (Insistent Fair Strategy Process). This algorithm uses the assessment of total bandwidth for customers. In general, DBA algorithms do not take into account type of services or overloaded ONUs. OLT grants more slots for heavy loaded ONU. In this point of view, IFAISTOS does not consider only each T-CONT by their priority; further, it considers the fair allocation for each ONU on ODN.

Furthermore, DBA algorithm with the consideration of "fairness" was proposed by Gravalos et al. [17]. Max-Min Fair DBA algorithm is applied to all ONUs with the "fairness" usage. Although DBA algorithms can change the minimum bandwidth allocation, it is still preserved and is guaranteed by T-CONT allocation. The authors have not presented results for full occupied OLT slots. It could prove the algorithm efficiency as well.

In general, more effort has been significantly put into EPON (Ethernet PON) networks and its algorithms. The results of [18] showed that it is not possible to directly use DBA for EPON in XG-PON because they exhibit the huge delay of services.

Researchers envisage using DBA algorithms with FTTA (Fiber to the Antenna) where an optical fiber is terminated at the base station of the cellular network, and then, the signal is spread by another technique [19]. GPON supports the protection scheme of active elements. However, ISPs do not have the effort to use it due to financial expenses. This protection schemes, let say approach, should be changed by XG-PON or NG-PON2 networks where the native support of DBA is presented, and ISPs should use some protection schemes because those networks might be subjected to attack or an emergency outage. Furthermore, each ISP may have various requirements (preferring strict priority, strict fairness, etc.). Three distinct scenarios are discussed in [20].

\section{DBA ALGORITHMS}

The main aim of DBA algorithm is to equitably divide bandwidth in the upstream direction for each ONU in PON. Downstream direction is broadcasted for all ONU and is processed by propriety ONU with exactly same parameters at ONU and in the frame. On the other hand, upstream direction is controlled by OLT with time slots. In other terms, TDM (Time Division Multiplex) divides bandwidth into the time slots, and they are allocated on demands for ONU. Time slots allocation is an objective of OLT unit, and it requires corresponding identifiers.

OLT uses BWmap (Bandwidth Map) part of each transferred frame where is specified: Alloc-ID, Start Time, Grant Size, DBRu (Dynamic Bandwidth Report) and other fields. AllocID identifiers ONU unit, Start Time is a time where ONU unit can start the transferring of data in the upstream direction, and
Grant size represents the total amount of data specific AllocID in the upstream direction and reports the requirements (data size) in next upstream frame. Concrete Alloc-ID and its offered load can be expressed by [6] Eq. 1 .

$$
R_{L}(t)=\frac{B(t)+A(t, t+\Delta)}{\Delta},
$$

where $B(t)$ is buffer occupation in time $t, \Delta$ is certain fixed time, and $A(t, t+\Delta)$ represents new incoming flow into buffer during the interval $t, t+\Delta$.

\section{A. Buffer reporting}

OLT has to ensure the fairness dynamic bandwidth allocation for the heavy load conditions for ONUs. In other words, if there are two ONUs and the first has the heavy loaded condition and the second does not have any data to transfer, then the OLT has to ensure more time slots for ONU1 than ONU2. The ONU periodically reports the occupation of the buffer to the OLT unit. The reporting can be realized in two ways:

1) Status reporting (SR).

2) Traffic reporting (TM).

The status reporting requires the cooperation of ONU and OLT because each ONU sends the information about own buffer occupation. This information is included in DBRu field of XGTC (Next Generation Transmission Convergence) frame. OLT divides time slots based on the information about a buffer occupation. The second method, traffic reporting, does not require such cooperation because OLT observes the data flows of ONU's units and grants the bandwidth by processing them.

If ONU does not have any data to process, the idle frame is sent. The first method requires more allocation of resources of OLT unit while SR has lower delay of processing. In general, SR is used together with DBA algorithms. However, modified Max-Min Fair DBA algorithm uses both methods (for more details see [17]).

\section{B. Round robin}

Round robin is a simple implementation of DBA algorithm for NS-3, especially the used one in XG-PON package. Round robin is based on one of the oldest, simplest and fairness algorithm. The main utilizing is in operational system but it can be used also in another part. The principle of the algorithm can be described as some amount of tasks. Each task runs one by one but only if it has a token of the cycle. In XG-PON package implementation, it refers to the fixed amount of bytes for each T-CONT during each cycle. The minimum time for each ONU queue processing can be expressed as:

$$
T=\frac{C}{N}
$$

where: $T$ means the time for each ONU/T-CONT processing (the ONU is able to send a data), $C$ represents the time of one cycle, and $N$ is the total number of ONUs. Disadvantage is a small value of $\mathrm{T}$ which leads to increase the time needed for processing by OLT. In other words, the time of simulation is increasing rapidly, especially, for a huge amount of ONUs. 


\section{GIANT algorithm}

The GIANT algorithm was developed and had been tested in a real environment in 2006. First of all, T-CONT (Transmission Container) has to be defined because GIANT algorithm uses it. T-CONT is a group of flows and it is meaningful in the upstream direction only and Alloc-ID exists only in namespace per PON while the external identifier of a T-CONT is hierarchical [21]. In general, there are four types of T-CONT divided as follow:

- T-CONT 1 - represents fixed bandwidth with the highest priority, for example for voice services. All ISPs guarantee a minimum bandwidth for customers in networks represented by T-CONT 1 .

- T-CONT 2 - assured bandwidth with the second higher priority (for example IPTV service).

- T-CONT 3 - non-assured bandwidth with high priority.

- T-CONT 4 - the best-effort bandwidth with the lowest priority FTP (File Transfer Protocol) or SNMP (Simple Network Management Protocol) etc.

GIANT DBA algorithm divides bandwidth in the first iteration by priority of T-CONTs. In the first step, the bandwidth for T-CONT 1 is assigned with each Alloc-ID. OLT does not consider buffer occupation of ONUs with T-CONT 1 bandwidth. The T-CONT 1 of the network in Slovakia has $64 \mathrm{kbit} / \mathrm{s}$ as a guarantee bandwidth for all customers. The assured bandwidth cannot exceed $R_{A}$ (in bit/s) and the nonassured $\left(R_{N A}\right)$ bandwidth is distributed to the depletion of the available bandwidth of T-CONTs. GIANT also uses SImax (Service Interval) and SImin for representation of a maximal and minimal service interval, respectively. These identifiers represent a requirement of bandwidth. Furthermore, the identifiers $A B \min$ and $A B$ sur represent Minimum Allocation Bytes and surplus allocation bytes, respectively.

GIANT dynamically determines $A B$ values and $S I \max$ for T-CONT 1, T-CONT 2, and interrogation T-CONT 4 and the assured path of T-CONT 3. Every $S I$ timer expiring causes reallocation of $A B$ for corresponding T-CONT. GIANT has been developed since 2006. Researchers expanded it by the $I A$ (Immediate Allocation) extension. The next modification came in 2013 and improved $I A$ 2008. It has allowed using the redundant bandwidth of one T-CONT and to allocate it in another one.

GIANT MAC (Medium Access Controller) makes decisions in the each cycle of the allocation process. T-CONT 1 does not use DBA because it has the guaranteed bandwidth in the initialization phase. ONU can add more than one Alloc-ID, if it adds a new one, then the controller checks available resources and verifies if the total allocated bandwidth does not overlap the total link capacity. Guarantee service speed equals $A B \min / S \operatorname{Im} a x$, where surplus capacity equals $A B$ sur/SImin. The sum of guaranteed and surplus capacity obtains total allowed bandwidth for T-CONT 3.

The allocation of surplus bandwidth is realized as follows; first of all is necessary to check if the processing of T-CONT is T-CONT 3 or 4 and if SImin timer is higher than 1. If it is, SImin timer is decremented by 1 . Moreover, if the number of frame's bytes is equal to zero so as to determine

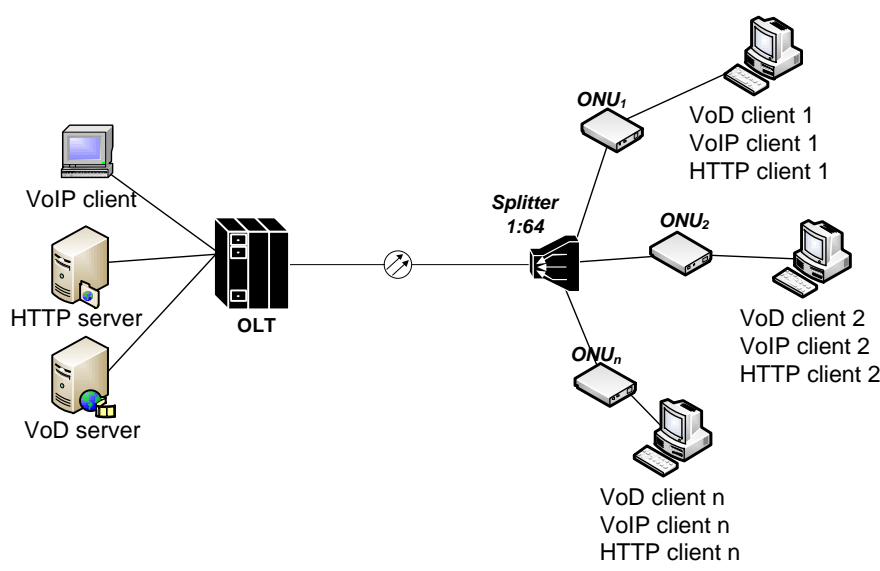

Fig. 2. Basic topology for simulation model of DBA algorithms

the start pointer T-CONT 3 such that the current indicator starts T-CONT 3 is added and subsequently sequence AllocID subtracting one. In other words, if the pointer of start is $N$ new value is $N+i$ where $i$ is iteration number. Next iteration checks again if next requirement is bigger than 0 and SImin equals 0 , than the surplus bandwidth is allocated.

First, we choose $L$ as a pointer to select the start of TCONT 4. The pointer of the start is equal to the sum of $L$ if the frame bytes are equal to zero and the order of current operation of all Alloc-ID is reduced by 1 . Otherwise, the frame of bytes is equal to zero and the next requirement is bigger than 1 and next SImin timer is equal to 0 . The surplus bandwidth is allocated in that manner.

\section{Simulation Model}

Our simulation model (see Fig. 2) contains OLT, ONUs, clients, and servers. NS-3 is not able to consider physical layer parameters (fiber length, split ratio, and various distances between OLT and ONU). That is why we had to include servers. These servers represent VoD (Video on Demand), HTTP (Hyper Text Transfer Protocol) servers, and VoIP (Voice over Internet Protocol) client.

The group of servers is interconnected with OLT via $1 \mathrm{Gbit} / \mathrm{s}$ line with $2 \mathrm{~ms}$ delay. ONU contains one PC (Personal Computer) which represents services consumption. ONU has the same line parameters due to the time complexity and hardware consumption of simulation. The data rate of VoD service was $10 \mathrm{Mbit} / \mathrm{s}$ and the OffTime was set-up as 0 (It means that the service is constantly up and running during the whole simulation scenario). The VoIP was set-up to standard data rate $64 \mathrm{kbit} / \mathrm{s}$, following the G.711 protocol. The HTTP traffic was generated by the HTTP server with the data rate $1 \mathrm{Mbit} / \mathrm{s}$.

\section{A. Results and discussion}

We have prepared two simulation scenarios. The first scenario included round robin DBA without any consideration of the dynamic reporting of buffer allocation. The second scenario included GIANT DBA algorithm. As a comparison, 
we performed two different simulations for each scenario, for 64 ONUs (it is ideal model for XG-PON single OLT port) and for an overloaded interface with connected 200 ONUs to this interface. These ONUs did not have real parameters because the split ratio depends on the parameter $n^{2}$ but our aim was to increase bandwidth consumption.

1) First scenario: The first scenario includes round robin DBA algorithm (we call it "algorithm without DBA") and unmodified GIANT DBA algorithm. All were applied for all services represented by T-CONTs. Our model consists of 3 services and a delay of traffic is observed. The delay of 64 ONUs is just $6-7 \mathrm{~ms}$ because the OLT unit is able to grant sufficient bandwidth for each one (see Fig. 3). The packet loss is equal to 0 and the total bandwidth is equal to the sum of services. Approximately constant delay of VoIP does not lead to any interference or echo during the call. The jitter of VoD is reduced by buffering service in ONU or set-top-box. Web browsing HTTP represents T-CONT 2 with higher priority.

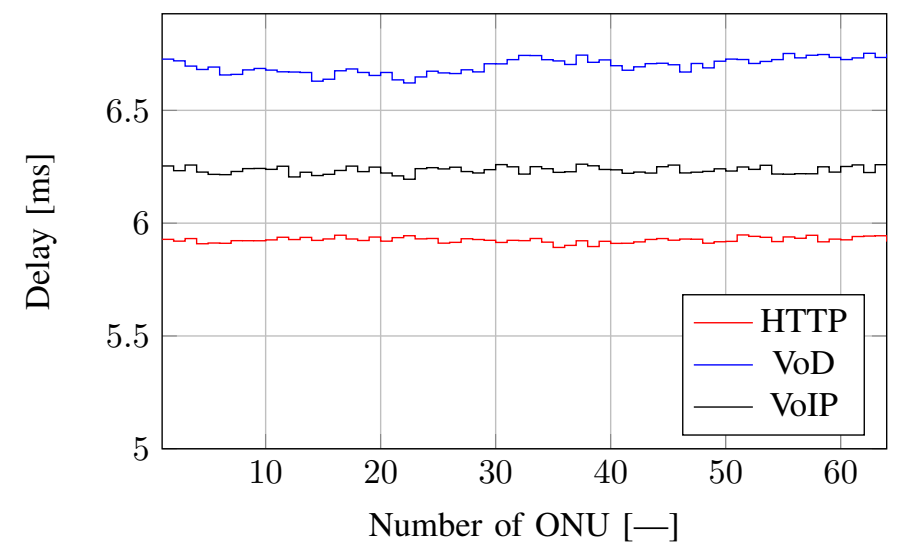

Fig. 3. Transmission delay without DBA algorithm for $64 \mathrm{ONU}$ units

Note that the NS-3 (Network Simulator) is not able to assign packets into real T-CONT by labeling packets in QoSTag library. QoSTag marks each packet with tag but our simulation labeling packets by IP address of nodes is used. Then one ONU contains another 3 nodes for representation separated services. Modified topology can be seen in Fig. 4.

We have again simulated 64 ONUs with GIANT DBA algorithm. The results of this simulation are shown in Fig. 5. As was expected GIANT DBA has better results and the delay for each ONU was lower. The significant mitigation had the VoD service, from $6.7 \mathrm{~ms}$ to $6.3 \mathrm{~ms}$ by GIANT algorithm

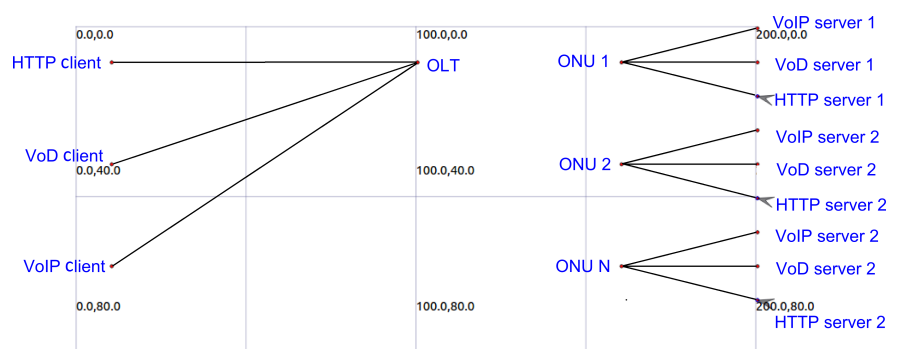

Fig. 4. Modified topology for GIANT algorithm simulations applied only for the OLT unit. The algorithm was modified due to NS-3 restrictions.

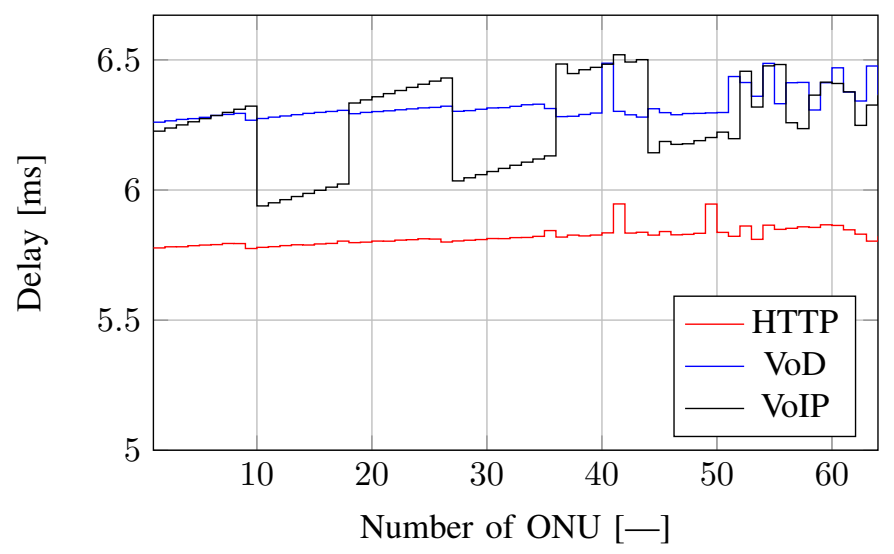

Fig. 5. Transmission delay with GIANT DBA algorithm for 64 ONU units

We compared a number of the packet drop during the simulation, without and with the DBA algorithm. As can be seen in Fig. 6 the difference is significant. The simulation without DBA round robin does not take into account any intelligence and fairness for the ONU traffic. The number of dropped packets was approximately 4000 packets for each ONU but in comparison with the unmodified GIANT algorithm, around 300 packets for several ONUs due to that the data of VoD were processed separately and in random sequence.

The ns-3 simulation tool uses a Callback for providing details of packets obtained such as a number of processed packets or dropped packets. After the Callback is configured, it can call an assigned function, which provides the results. During this function, it is possible to create computations or records of running events. For example, the ns-3 simulation tool can call the function anytime when the packet is dropped due to a stack overflow. On the other hand, callback works only for P2P links. However, the XG-PON package for ns3 does not contain an implementation of Callback. That is the reason why it is not possible to obtain more details about dropped packets etc. On the other hand, we know sending and receiving time of each packet because all data are stored in a set. The client is able to calculate a delay value for each packet. First of all, values with receiving and sending time are discovered and deducted, after that, the values are removed from the set.

2) Second scenario: In the second scenario, our modified GIANT algorithm was used and compared with unmodified GIANT DBA. The topology was same and contained: one OLT, 64 ONUs (each one has 3 services - data, video, and voice). Modified algorithm first checks buffer occupancy of T-CONT and based on T-CONT type allocates adequate bandwidth. If the buffer occupation for assured bandwidth is bigger than 1 , then there are three conditions:

1) If the buffer occupancy is less than 4 , then allocated value is equal to 4 .

2) OLT checks BWmap (a database with history of BWmap allocation is used) if the ONU gave some allocations pre- 


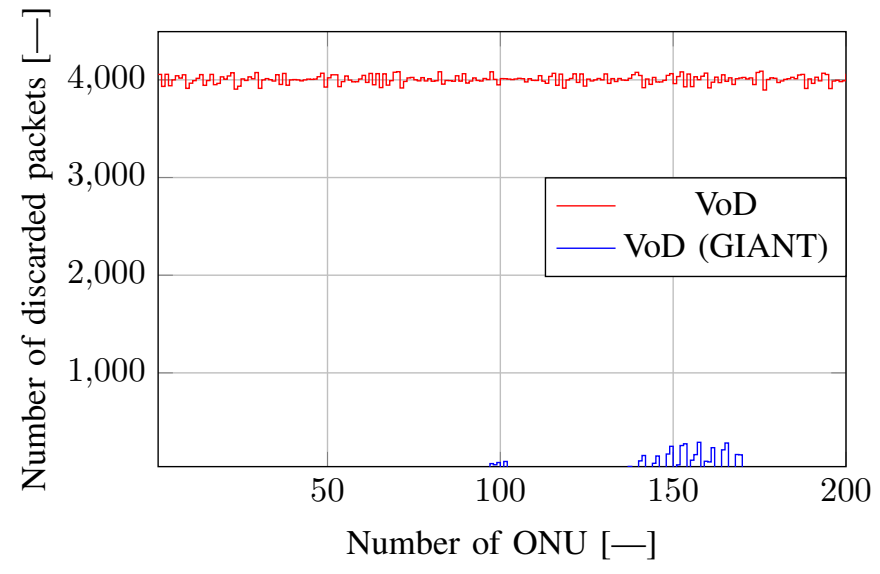

Fig. 6. The number of discards packet for XG-PON network with 200 ONUs with/without DBA

viously. If yes, the buffer occupancy value is increased by 1 . Or,

3) Or this condition tests whether the allocated bandwidth is equal to allocated bytes.

In general, those conditions are tested only if the timer of the type T-CONT expired (equals 0), if yes, then those conditions are tested. If not, the algorithm does not test conditions. In other words, T-CONT does not have urgent data and in next round is decremented by 1 .

We removed checking of T-CONT timers which leads to increasing control data but also to decreasing delay of services. We tested services together but graphs are separated due to lucidity. As can be seen in Fig. 7, the delay of HTTP service is reduced by $0.8 \mathrm{~ms}$ in comparison with the simulation without DBA and by $0.3 \mathrm{~ms}$ according to unmodified GIANT, respectively. Next service is $\mathrm{VoD}$ with $6.6 \mathrm{~ms}$ delay with DBA algorithm and average in $6.4 \mathrm{~ms}$ delay with the unmodified GIANT algorithm (see Fig. 8). Finally, by our modification, we reduced delay by $0.35 \mathrm{~ms}$ for each ONU on ODN. For the last one service (VoIP) delay was reduced around $0.2 \mathrm{~ms}$ although VoIP uses T-CONT 1 which has the highest priority and DBA does not consider it (see Fig. 9).

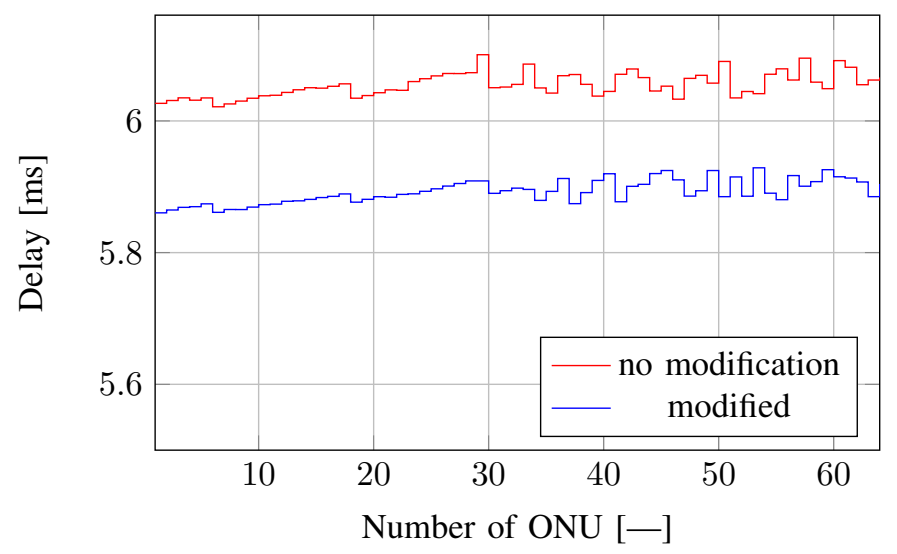

Fig. 7. Transmission delay of HTTP service for 64 ONUs with unmodified and modified GIANT algorithm

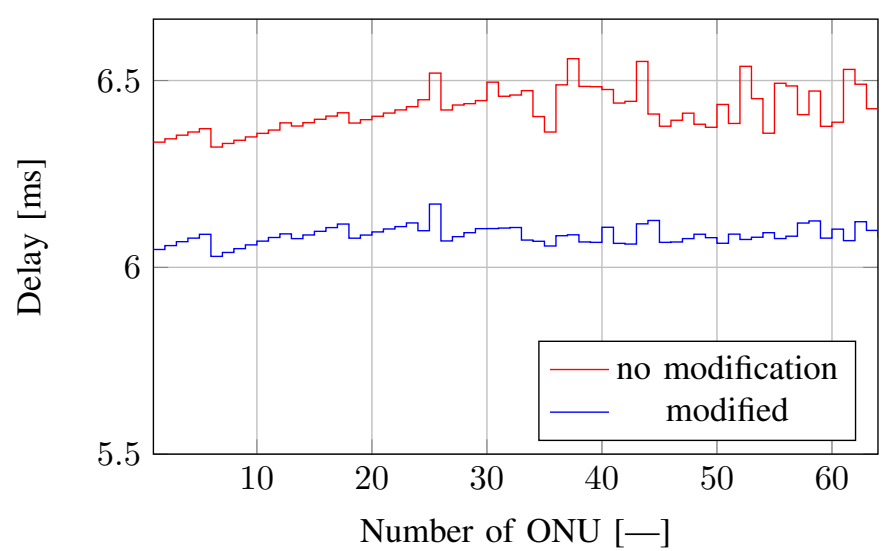

Fig. 8. Transmission delay of VoD service for 64 ONUs with unmodified and modified GIANT algorithm

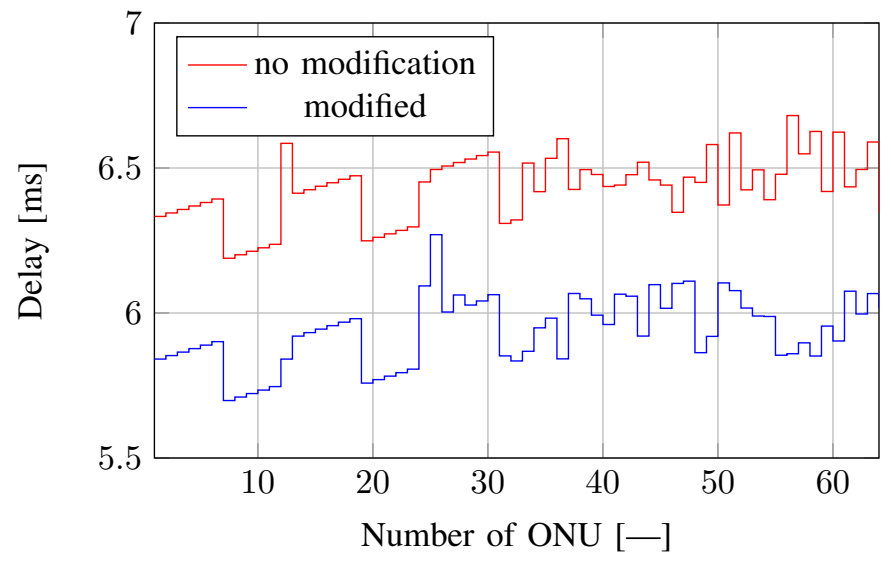

Fig. 9. Transmission delay of VoIP service for 64 ONUs with unmodified and modified GIANT algorithm

\section{CONCLUSION}

XG-PON standard is the most promising solution for migration from GPON but we cannot expect it in near future due to maturity of GPON and price of elements. XG-PON systems also will need cooling because the downstream wavelength range is very narrow and it will lead to increasing price of the system. On the other hand, video streaming service such as Netflix (4k video) etc. requires bigger bandwidth than standard SD (Standard Definition) video broadcasted by IPTV. GPON networks support only lower split ratio but an optical amplifier can reduce this limitation. However, the ODN will not stay passive.

In this article, we presented XG-PON GIANT dynamic bandwidth allocation algorithm and proposed own improvement due to the reduction of delay for services. The delay was reduced for each service but VoIP service used the T-CONT 1 with the highest priority and the bandwidth is allocated for each ONU. We can also describe this T-CONT as guaranteed bandwidth at ONU because OLT serves this T-CONT for each ONU. By our modification in the GIANT algorithm, we reduced delay around $0.3 \mathrm{~ms}$ for HTTP service and about $0.35 \mathrm{~ms}$ for VoD service, respectively.

The future research will continue with implementation of another DBA algorithm into our simulation model and testing 
algorithms on a real optical network. We would like to add a Callback function which will be able to provide details of dropped packets.

\section{ACKNOWLEDGMENT}

Presented research has been supported by the project: E infrastructure CESNET - modernization, reg. nr. CZ.02.1.01/0.0/0.0/16_013/000179.

\section{REFERENCES}

[1] The Zettabyte EraTrends and Analysis, 2016. [Online]. Available: goo.gl/dAO7EY.

[2] Next Generation Access Networks (NGA), EUR Lex: Access to European Union law, 2010. [Online]. Available: http://eurlex.europa.eu/legal-content/EN/TXT/?uri=URISERV\%3Asi0018.

[3] N. Angelopoulos, P. Solina, and J. D. Angelopoulos, The IST-GIANT project (GIgaPON Access NeTwork), in Proceedings of the 7th European Conference on Networks and Optical Communications, 2002 p. 119-126.

[4] G.987.1 : 10-Gigabit-capable passive optical networks (XG-PON) General requirements, International Telecommunication Union, 2016. Available: https://www.itu.int/rec/T-REC-G.987.1/en.

[5] G.987.2 : 10-Gigabit-capable passive optical networks (XG-PON): Physical media dependent (PMD) layer specification, International Telecommunication Union, 2016. Available: https://www.itu.int/rec/TREC-G.987.2/en

[6] G.987.3 : 10-Gigabit-capable passive optical networks (XG-PON): Transmission convergence (TC) layer specification, International Telecommunication Union, 2014. Available: https://www.itu.int/rec/TREC-G.987.3/en.

[7] Next-Generation PON Evolution - Huawei, Huawe Technologies Co., Ltd., c2016. [Online]. Available: www.huawei.com/ilink/en/download/HW_077443.

[8] T. F. Fent, S. Shaari, and B. Y. Majlis, Distributed CATV Inputs in FTTH-PON System, in 2006 IEEE International Conference on Semiconductor Electronics, 2006, pp. 58-61.

[9] J. Li, H. He, and W. Hu, Impact of TWDM on CATV and fronthaul channels, in 2015 14th International Conference on Optical Communications and Networks (ICOCN), 2015, pp. 1-3.

[10] C. Antony, M. D. Santa, G. Talli, and P. D. Townsend, Design and Performance Analysis of Raman Amplified XG-PON System, Journal of Lightwave Technology, vol. 34, no. 11, pp. 2692-2701, 2016.

[11] R. S. Penze, J. B. Rosolem, U. R. Duarte, G. E. R. Paiva, and R. B. Filho, Fiber Powered Extender for XG-PON/G-PON Applications, Journal of Optical Communications and Networking, vol. 6, no. 3, p. 250-258, 2014.

[12] J. A. Arokkiam, K. N. Brown, and C. J. Sreenan, Refining the GIANT dynamic bandwidth allocation mechanism for XG-PON, in 2015 IEEE International Conference on Communications (ICC), 2015, pp. 10061011.

[13] C. A. Kyriakopoulos and G. I. Papadimitriou, Bandwidth efficiency in the next generation access architecture XG-PON, in 2016 Eighth International Conference on Ubiquitous and Future Networks (ICUFN), 2016, pp. 833-838.

[14] M. -S. Han, Simple and feasible dynamic bandwidth and polling allocation for XGPON, in 16th International Conference on Advanced Communication Technology, 2014, pp. 298-304.

[15] P. Sarigiannidis, D. Pliatsios, T. Zygiridis, and N. Kantartzis, DAMA A data mining forecasting DBA scheme for XG-PONs, in 2016 th International Conference on Modern Circuits and Systems Technologies (MOCAST), 2016, pp. 1-4.

[16] P. Sarigiannidis, G. Papadimitriou, P. Nicopolitidis, E. Varvarigos, M Louta, and V. Kakali, IFAISTOS: A fair and flexible resource allocation policy for next-generation passive optical networks, in 2014 6th International Congress on Ultra Modern Telecommunications and Control Systems and Workshops (ICUMT), 2014, pp. 7-14.

[17] I. Gravalos, K. Yiannopoulos, G. Papadimitriou, and E. A. Varvarigos, A modified max-min fair dynamic bandwidth allocation algorithm for XG-PONs, in 2014 19th European Conference on Networks and Optical Communications - (NOC), 2014, pp. 57-62.
[18] F. Aurzada, M. Scheutzow, M. Reisslein, N. Ghazisaidi, and M. Maier, Capacity and Delay Analysis of Next-Generation Passive Optical Networks (NG-PONs), IEEE Transactions on Communications, vol. 59, no. 5, pp. 1378-1388, 2011.

[19] J. A. Arokkiam, K. N. Brown, and C. J. Sreenan, Optimised QoS-Aware DBA Mechanisms in XG-PON for Upstream Traffic in LTE Backhaul, in 2016 IEEE 4th International Conference on Future Internet of Things and Cloud Workshops (FiCloudW), 2016, pp. 361-368.

[20] C. Li, W. Guo, W. Wang, W. Hu, and M. Xia, Bandwidth Resource Sharing on the XG-PON Transmission Convergence Layer in a Multioperator Scenario, Journal of Optical Communications and Networking, vol. 8 , no. 11 , p. 835-843, 2016.

[21] D. Hood and E. Trojer, Gigabit-capable passive optical networks. Hoboken: Wiley, 2011

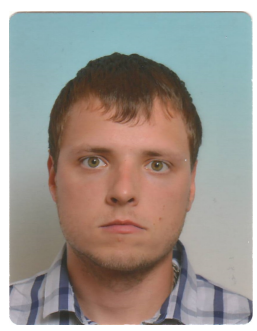

Tomas Horvath (MSc) was born in Havirov, Czech Republic in 1989. He received his MSc. degrees in Telecommunications from the Brno University of Technology, Brno, in 2013. His research interests include passive optical networks (xPON), optoelectronics, and fiber-optic telecommunications. Currently, he has been actually post graduate student at Brno University of Technology, Department of Telecommunications and his topic of dissertation thesis is Optimization services in FTTx optical access networks.

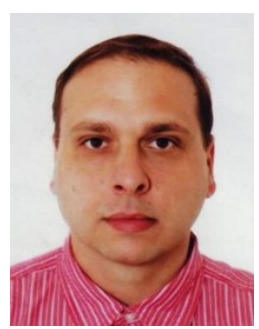

Vaclav Oujezsky (MSc) was born in Brno, Czech Republic. Post graduate student at Brno University of Technology, Department of Telecommunications, Senior Network Engineer at T-Mobile CZ, and currently at IBM CZ. Working actively on projects of security and transport networks at laboratory SIX His research interests include implementation of evolutionary algorithm, Cisco, Python, VHDL, and converged networks. His topic of dissertation thesis is Converged Networks and Traffic Tomography by Using Evolutionary Algorithms.

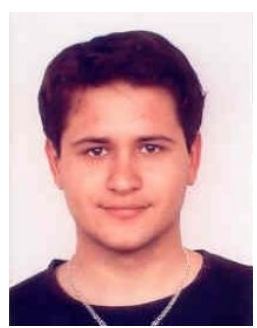

Petr Munster (MSc, Ph.D.) was born in 1984,in Zlin (Czech Republic). He received his $\mathrm{PhD}$ at the Brno University of Technology, Department of Telecommunications in 2014 on the thesis entitled Parameters of the FTTx networks. His current research themes focus on fiber-optic sensors, especially distributed fiber-optic sensors, and also on fiber-optic telecommunications. He has about 50 scientific publications in journals and conferences in last 5 years.

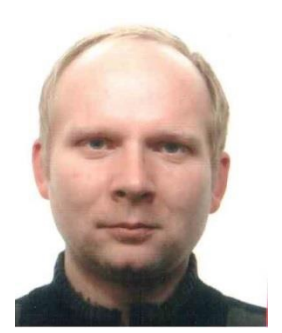

Josef Vojtech is a head of research department of Optical networks at CESNET a.l.e., operator of national research and education e-infrastructure of the Czech Republic. He received his MS and BS degrees in computer science and pedagogy from the Czech Technical University in 2001 and 2003 respectively, and his $\mathrm{PhD}$ degree in field of optical networking from the Czech Technical University in 2008. His record shows more than 65 peer reviewed proceedings and journal papers. He holds 18 patents (including US and EU) and utility models. He participated in international projects: COMPLETE, FI-PPP XIFI, GN4, GN3+ GN3, GN2, Porta Optica Study and SEEFIRE. His current research interests include software defined optical networking and special photonic services as precise time, stable frequency or sensing. He is a member of SPIE, IEEE and OSA. In 2007 he received the Research prize of the Czech minister of education. 


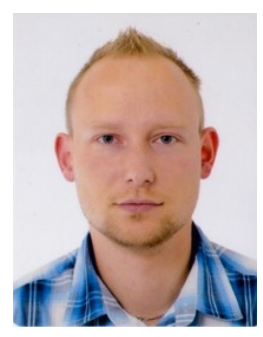

Ondrej Havlis received the Master's degree in the field communications and Informatics science from the Brno University of Technology, in 2012. He joined Optical networks department of CESNET, a.1.e., in 2012. He is active in research, design and verification of Photonic Services in the optical networks and development Photonic Testbed. He was actively involved in the project GN3 and GN3plus. In 2015 Ondrej has started to study at Brno University of Technology at Faculty of Electrical Engineering and Communication PhD degree with topic of this name Effective Optical Function in Fibre Photonic Networks. Currently Ondrej participates in the project threat detection of infrastructure safety.

Pavel Sikora (BCS) is a student at Brno University of Technology in master degree. His master's thesis deals with dynamic bandwidth allocation algorithms and theirs testing at low cost devices. 